Article

\title{
Enantioselective synthesis of chiral phosphonylated 2,3-dihydrofurans by copper catalyzed asymmetric formal [3+2] cycloaddition of propargylic esters with $\beta$-keto phosphonates
}

\author{
Xiushuai Chen a,b, Chuanjin Hou ${ }^{a, *}$, Qing Li a,b, Yanjun Liu a,\#, Ruifeng Yang a , Xiangping Hu ${ }^{\text {b,\$ }}$ \\ a School of Light Industry and Chemical Engineering, Dalian Polytechnic University, Dalian 116034, Liaoning, China \\ b Dalian Institute of Chemical Physics, Chinese Academy of Sciences, Dalian 116023, Liaoning, China
}

A R T I C L E I N F O

Article history:

Received 21 May 2016

Accepted 14 June 2016

Published 5 August 2016

Keywords:

Copper

Asymmetric synthesis

[3+2] Cycloaddition

$\beta$-Keto phosphonates

Phosphonylated 2,3-dihydrofurans

\begin{abstract}
A B S T R A C T
Copper catalyzed asymmetric formal [3+2] cycloaddition of propargylic esters to $\beta$-keto phosphonates for the synthesis of chiral phosphonylated 2,3-dihydrofurans was developed. By using a bulky and structurally rigid tridentate ketimine $\mathrm{P}, \mathrm{N}, \mathrm{N}$ ligand, a series of optically active phosphonylated 2,3-dihydrofurans were prepared in high yield and up to $92 \%$ ee.
\end{abstract}

(c) 2016, Dalian Institute of Chemical Physics, Chinese Academy of Sciences. Published by Elsevier B.V. All rights reserved.

\section{Introduction}

Enantiomerically enriched 2,3-dihydrofuran derivatives are very important compounds which are found in a variety of natural products and biological molecules [1,2]. They are also convenient precursors for the asymmetric synthesis of tetrahydrofurans [3-5]. Thus, much effort have been devoted to developing efficient methods for the synthesis of chiral 2,3-dihydrofurans, such as the organocatalytic domino $\mathrm{Mi}$ chael-alkylation reaction [6-8], interrupted Feist-Bénary reaction $[9,10]$ or modified Feist-Bénary reaction [11]. In addition, the transition metal catalyzed asymmetric synthesis of chiral 2,3-dihydrofurans has also attracted much attention in the past few decades. Ozawa et al. [12] obtained chiral 2-aryl-2,3-dihy- drofurans by Pd catalyzed asymmetric arylation of 2,3-dihydrofuran involving a kinetic resolution process. Evans et al [13] developed a Sc catalyzed [3+2] cycloaddition of allenylsilanes with ethyl glyoxylate for the synthesis of chiral 2,3-dihydrofurans. Recently, Son et al. [14] and Zhou et al. [15] reported the enantioselective synthesis of chiral 2,3-dihydrofurans by $\mathrm{Cu}$ catalyzed asymmetric [4+1] cycloaddition of enones with diazo compounds. Despite these advances, the development of new catalysts for the enantioselective synthesis of chiral 2,3-dihydrofurans is still in demand.

Following the pioneering works of Nishibayashi et al. [16] and van Maarseveen et al. [17], $\mathrm{Cu}$ catalyzed asymmetric propargylic transformation has made significant progress [18-21]. Recently, we have developed a series of chiral tridentate

\footnotetext{
*Corresponding author. E-mail: houcj@dlpu.edu.cn

\# Corresponding author. E-mail: yjliu65@sohu.com

\$Corresponding author. E-mail: xiangping@dicp.ac.cn

This work was supported by the National Natural Science Foundation of China $(21403022,21572226)$ and the Natural Science Foundation of Liaoning Province of China (2015020194).

DOI: 10.1016/S1872-2067(16)62488-9 | http://www.sciencedirect.com/science/journal/18722067 | Chin. J. Catal., Vol. 37, No. 8, August 2016
} 
$\mathrm{P}, \mathrm{N}, \mathrm{N}$-ligands which showed excellent diastereo- and enantioselectivity in the Cu catalyzed asymmetric propargylic substitution [22-26], decarboxylative propargylic substitution [27-29], [3+3] cycloaddition [30], [3+2] cycloaddition [31,32] and [4+2] cycloaddition [33]. In particular, we reported an example of $\mathrm{Cu}$ catalyzed formal [3+2] cycloadditon of propargylic esters with $\beta$-ketoesters for the enantioselective synthesis of chiral 2,3-dihydrofurans [31]. Considering the important biological activity of phosphonylated heterocyclic compounds, we envisioned that this strategy is also suitable for the synthesis of chiral phosphonylated 2,3-dihydrofurans if a $\beta$-keto phosphonate is employed as the substrate instead of $\beta$-ketoester. As a result, here we report the $\mathrm{Cu}$ catalyzed formal [3+2] cycloadditon of propargylic esters with $\beta$-keto phosphonates for the synthesis of chiral phosphonylated 2,3-dihydrofurans with a bulky and structurally rigid tridentate ketamine $\mathrm{P}, \mathrm{N}, \mathrm{N}$ ligand.

\section{Experimental}

\subsection{General}

All reactions were carried out under a nitrogen atmosphere using standard Schlenk techniques. All solvents were purified by standard procedures and stored under nitrogen. ${ }^{1} \mathrm{H}$ NMR and ${ }^{13} \mathrm{C}$ NMR spectra were recorded at $400 \mathrm{MHz}$ and $100 \mathrm{MHz}$, respectively, on a Bruker DPX400 NMR spectrometer (Bruker, Switzerland). Enantiomeric ratios were determined by chiral HPLC using $n$-hexane and $i$-PrOH as the mobile phases. Optical rotations were recorded on a JASCO P-1020 polarimeter (JASCO Corporation, Tokyo, Japan).

\subsection{General procedure for Cu catalyzed formal [3+2] cycloaddition of propargylic esters with $\beta$-keto phosphonates}

$\mathrm{Cu}(\mathrm{OTf})_{2}(5.4 \mathrm{mg}, 0.015 \mathrm{mmol})$ and $(S)-\mathbf{L} 4 \mathbf{b}(7.8 \mathrm{mg}, 0.0165$ $\mathrm{mmol})$ were added to anhydrous methanol $(1 \mathrm{~mL})$. The resulting mixture was stirred at room temperature under a nitrogen atmosphere for $1 \mathrm{~h}$. Then, a solution of propargylic ester 1 (0.6 $\mathrm{mmol})$ and $\beta$-keto phosphonate $2(0.3 \mathrm{mmol})$ in $2 \mathrm{~mL}$ of anhydrous methanol was added. The resulting mixture was stirred at $-20{ }^{\circ} \mathrm{C}$ for $24 \mathrm{~h}$. The reaction mixture was then concentrated under vacuum and the residue was purified by silica gel chromatography to afford the corresponding chiral phosphonylated 2,3-dihydrofurans 3.

(-)-Dimethyl(5-methylene-2,4-diphenyl-4,5-dihydrofuran-3yl)phosphonate (3aa). A colorless oil was obtained in $88 \%$ yield after purification with column chromatography on silica gel (hexane/ethyl acetate, 4:1-1:1). 90\% ee was determined by chiral HPLC (Chiralcel OJ-H, $n$-hexane $/ i$-PrOH $=90 / 10,0.8$ $\left.\mathrm{mL} / \mathrm{min}, 254 \mathrm{~nm}, 40^{\circ} \mathrm{C}\right): t_{\mathrm{R}}$ (major) $=14.5 \mathrm{~min}, t_{\mathrm{R}}($ minor $)=8.7$ min. $\left.[\alpha]_{\mathrm{D}}{ }^{22}=-84.9(c) 1.13, \mathrm{CH}_{2} \mathrm{Cl}_{2}\right) .{ }^{1} \mathrm{H}$ NMR $(400 \mathrm{MHz}$, DMSO- $\left.d_{6}\right): \delta 7.91(\mathrm{~d}, J=7.5 \mathrm{~Hz}, 2 \mathrm{H}), 7.58-7.50(\mathrm{~m}, 3 \mathrm{H})$, 7.41-7.27 (m, 5H), 5.12-5.03 (m, 1H), 4.85-4.73 (m, 1H), 4.20 (s, $1 \mathrm{H}), 3.39$ (d, $J=3.6 \mathrm{~Hz}, 3 \mathrm{H}), 3.21$ (d, $J=11.3 \mathrm{~Hz}, 3 \mathrm{H}) ;{ }^{13} \mathrm{C}$ NMR (100 MHz, DMSO- $d_{6}$ ): $\delta 164.4$ (d, $J=13.9 \mathrm{~Hz}$ ), 163.5 (d, $J=$ $25.7 \mathrm{~Hz}), 142.6,131.6,129.1,129.0,128.7,128.6,128.2,127.7$,
102.7 (d, $J=214.2 \mathrm{~Hz}$ ), 88.0, 54.7 (d, $J=10.1 \mathrm{~Hz}$ ), 52.5 (d, $J=5.7$ $\mathrm{Hz}$ ), 52.3 (d, $J=5.5 \mathrm{~Hz}$ ); ${ }^{31} \mathrm{P}$ NMR (162 MHz, DMSO- $d_{6}$ ): $\delta 16.5$; HRMS calc. for $\mathrm{C}_{19} \mathrm{H}_{20} \mathrm{O}_{4} \mathrm{P}[\mathrm{M}+\mathrm{H}]^{+}: 343.1099$, found: 343.1094 .

${ }^{1} \mathrm{H}$ NMR (400 MHz, $\left.\mathrm{CDCl}_{3}\right): \delta 7.93(\mathrm{~d}, J=8.0 \mathrm{~Hz}, 2 \mathrm{H})$, 7.50-7.39 (m, 7H), 5.16 (d, $J=2.2 \mathrm{~Hz}, 1 \mathrm{H}), 4.88$ (d, $J=2.3 \mathrm{~Hz}$, 1H), 4.31 (s, 1H), 3.49 (d, $J=11.3 \mathrm{~Hz}, 3 \mathrm{H}), 3.33$ (d, $J=11.3 \mathrm{~Hz}$, $3 \mathrm{H})$.

(-)-Dimethyl (5-methylene-4-phenyl-2-( $p$-tolyl)-4,5-dihydrofuran-3-yl)phosphonae (3ba). A colorless oil was obtained in $79 \%$ yield after purification with column chromatography on silica gel (hexane/ethyl acetate, 4:1-1:1). 89\% ee was determined by chiral HPLC (Chiralcel $0 \mathrm{~J}-\mathrm{H}, n$-hexane $/ i-\mathrm{PrOH}=$ 90/10, $\left.0.8 \mathrm{~mL} / \mathrm{min}, 254 \mathrm{~nm}, 40{ }^{\circ} \mathrm{C}\right): t_{\mathrm{R}}$ (major) $=10.6 \mathrm{~min}, t_{\mathrm{R}}$ (minor) $=7.9 \mathrm{~min} .[\alpha]_{\mathrm{D}}{ }^{21}=-89.2\left(c 1.12, \mathrm{CH}_{2} \mathrm{Cl}_{2}\right) .{ }^{1} \mathrm{H}$ NMR $(400$ MHz, DMSO- $\left.d_{6}\right): \delta 7.76(\mathrm{~d}, J=8.0 \mathrm{~Hz}, 2 \mathrm{H}), 7.33-7.22(\mathrm{~m}, 7 \mathrm{H})$, 5.02-4.95 (m, 1H), 4.72-4.68 (m, 1H), $4.13(\mathrm{~s}, 1 \mathrm{H}), 3.32(\mathrm{~d}, J=$ $11.3 \mathrm{~Hz}, 3 \mathrm{H}$ ), 3.16 (d, $J=11.3 \mathrm{~Hz}, 3 \mathrm{H}$ ), 2.33 (s, 3H); ${ }^{13} \mathrm{C} \mathrm{NMR}$ (100 MHz, DMSO- $d 6$ ): $\delta 169.2$ (d, $J=13.9 \mathrm{~Hz}), 168.3$ (d, $J=26.0$ $\mathrm{Hz}), 147.5,146.4,134.1,133.8,133.7,132.9,132.4,130.5,106.6$ (d, $J=214.3 \mathrm{~Hz}), 92.6,59.5(\mathrm{~d}, J=10.1 \mathrm{~Hz}), 57.2(\mathrm{~d}, J=5.8 \mathrm{~Hz}$ ), 57.0 (d, $J=5.5 \mathrm{~Hz}$ ), 26.3; ${ }^{31} \mathrm{P}$ NMR (162 MHz, DMSO- $\left.d_{6}\right): \delta 21.6$; HRMS calc. for $\mathrm{C}_{20} \mathrm{H}_{22} \mathrm{O}_{4} \mathrm{P}[\mathrm{M}+\mathrm{H}]+$ : 357.1256, found: 357.1253.

(-)-Dimethyl (5-methylene-4-phenyl-2-( $m$-tolyl)-4,5-dihydrofuran-3-yl)phosphonate (3ca). A colorless oil was obtained in $84 \%$ yield after purification with column chromatography on silica gel (hexane/ethyl acetate, 4:1-1:1). 90\% ee was determined by chiral HPLC (Chiralcel OJ-H, $n$-hexane $/ \mathrm{i}-\mathrm{PrOH}=$ 90/10, $0.8 \mathrm{~mL} / \mathrm{min}, 254 \mathrm{~nm}, 40{ }^{\circ} \mathrm{C}$ ): $t_{\mathrm{R}}$ (major) $=10.5 \mathrm{~min}, t_{\mathrm{R}}$ (minor) $=7.3 \mathrm{~min} .[\alpha]_{\mathrm{D}}{ }^{22}=-81.3\left(c 1.10, \mathrm{CH}_{2} \mathrm{Cl}_{2}\right) .{ }^{1} \mathrm{H}$ NMR $(400$ MHz, DMSO- $\left.d_{6}\right): \delta 7.75-7.69(\mathrm{~m}, 2 \mathrm{H}), 7.40-7.28(\mathrm{~m}, 7.3 \mathrm{~Hz}, 7 \mathrm{H})$, 5.07-5.02 (m, 1H), 4.79-4.75 (m,1H), $4.19(\mathrm{~s}, 1 \mathrm{H}), 3.37(\mathrm{~d}, J=$ $11.3 \mathrm{~Hz}, 3 \mathrm{H}), 3.21$ (d, $J=11.3 \mathrm{~Hz}, 3 \mathrm{H}), 2.38$ (s, 3H); ${ }^{13} \mathrm{C}$ NMR (100 MHz, DMSO-d6): $\delta 164.4(\mathrm{~d}, J=14.0 \mathrm{~Hz}), 163.6$ (d, $J=25.8$ $\mathrm{Hz})$, 142.7, 138.0, 132.2, 129.3, 129.1, 128.6, 128.5, 128.2, 127.7, 126.3, 102.6 (d, $J=214.7 \mathrm{~Hz}), 87.9,54.7$ (d, $J=10.1 \mathrm{~Hz}$ ), $52.5(\mathrm{~d}, J=5.8 \mathrm{~Hz}), 52.3(\mathrm{~d}, J=5.5 \mathrm{~Hz}), 21.4 ;{ }^{31} \mathrm{P} \mathrm{NMR}(162 \mathrm{MHz}$, DMSO- $d_{6}$ ): $\delta$ 16.6; HRMS calc. for $\mathrm{C}_{20} \mathrm{H}_{22} \mathrm{O}_{4} \mathrm{P}[\mathrm{M}+\mathrm{H}]^{+}:$357.1256, found: 357.1252 .

(-)-Dimethyl (5-methylene-4-phenyl-2-(o-tolyl)-4,5-dihydrofuran-3-yl)phosphonae (3da). A colorless oil was obtained in $61 \%$ yield after purification with column chromatography on silica gel (hexane/ethyl acetate, 4:1-1:1). 88\% ee was determined by chiral HPLC (Chiralcel $0 \mathrm{~J}-\mathrm{H}, n$-hexane $/ i$-PrOH $=$ 90/10, $0.8 \mathrm{~mL} / \mathrm{min}, 254 \mathrm{~nm}, 40{ }^{\circ} \mathrm{C}$ ): $t_{\mathrm{R}}$ (major) $=14.2 \mathrm{~min}, t_{\mathrm{R}}$ (minor) $=9.1 \mathrm{~min} .[\alpha]_{\mathrm{D}}{ }^{21}=-89.2\left(c 1.10, \mathrm{CH}_{2} \mathrm{Cl}_{2}\right) .{ }^{1} \mathrm{H}$ NMR $(400$ MHz, DMSO- $\left.d_{6}\right): \delta 7.48(\mathrm{~d}, J=7.5 \mathrm{~Hz}, 1 \mathrm{H}), 7.42-7.29(\mathrm{~m}, 8 \mathrm{H})$, 5.08-5.02 (m, 1H), 4.76-4.70 (m, 1H), $4.17(\mathrm{~s}, 1 \mathrm{H}), 3.26(\mathrm{~d}, J=$ $11.3 \mathrm{~Hz}, 3 \mathrm{H}$ ), 3.12 (d, $J=11.3 \mathrm{~Hz}, 3 \mathrm{H}), 2.40$ (s, 3H); ${ }^{13} \mathrm{C} \mathrm{NMR}$ (100 MHz, DMSO- $d_{6}$ ): $\delta 165.3(\mathrm{~d}, J=3.3 \mathrm{~Hz}$ ), 165.1 (d, $J=15.2$ $\mathrm{Hz}), 142.3,137.1,130.8,130.6,130.4,129.4,129.1,128.3$, 127.7, 125.8, 105.4 (d, $J=214.7 \mathrm{~Hz}), 88.0,53.6$ (d, $J=10.5 \mathrm{~Hz}$ ), $52.2(\mathrm{~d}, J=5.6 \mathrm{~Hz}), 52.0$ (d, $J=5.4 \mathrm{~Hz}), 19.8$; ${ }^{31} \mathrm{P}$ NMR $(162 \mathrm{MHz}$, DMSO- $d_{6}$ ): $\delta$ 15.4; HRMS calc. for $\mathrm{C}_{20} \mathrm{H}_{22} \mathrm{O}_{4} \mathrm{P}[\mathrm{M}+\mathrm{H}]^{+}: 357.1256$, found: 357.1258 .

(-)-Dimethyl (2-(2-bromophenyl)-5-methylene-4-phenyl4,5-dihydrofuran-3-yl)phosphonate (3ea). A colorless oil was obtained in $68 \%$ yield after purification with column chroma- 
tography on silica gel (hexane/ethyl acetate, 4:1-1:1). 92\% ee was determined by chiral HPLC (Chiralcel OJ-H, $n$-hexane/ $i-\mathrm{PrOH}=80 / 20,0.8 \mathrm{~mL} / \mathrm{min}, 254 \mathrm{~nm}, 40{ }^{\circ} \mathrm{C}$ ): $t_{\mathrm{R}}$ (major) $=13.2$ $\min , t_{\mathrm{R}}$ (minor) $=9.1 \mathrm{~min} .[\alpha]_{\mathrm{D}}{ }^{24}=-92.8\left(c 1.30, \mathrm{CH}_{2} \mathrm{Cl}_{2}\right) \cdot{ }^{1} \mathrm{H}$ NMR (400 MHz, DMSO- $d_{6}$ ): $\delta 7.77(\mathrm{~d}, J=7.7 \mathrm{~Hz}, 1 \mathrm{H}), 7.62-7.60$ (m, 1H), 7.53-7.30 (m, 7H), 5.07-5.02 (m, 1H), 4.77-4.73 (m, $1 \mathrm{H}), 4.18$ (s, 1H), 3.35 (d, $J=11.3 \mathrm{~Hz}, 3 \mathrm{H}), 3.13$ (d, $J=11.3 \mathrm{~Hz}$, $3 \mathrm{H}) ;{ }^{13} \mathrm{C}$ NMR (100 MHz, DMSO- $\left.d_{6}\right): \delta 165.3(\mathrm{~d}, J=13.9 \mathrm{~Hz}$ ), $163.8(\mathrm{~d}, J=25.4 \mathrm{~Hz}), 142.0,132.9,132.7,132.6,131.2,129.0$, $128.6,127.9,127.7,122.4,106.4$ (d, $J=212.8 \mathrm{~Hz}$ ), 88.4, 53.4 (d, $J=10.1 \mathrm{~Hz}$ ), 52.3 (d, $J=5.4 \mathrm{~Hz}), 52.2\left(\mathrm{~d}, J=5.2 \mathrm{~Hz}\right.$ ); ${ }^{31} \mathrm{P}$ NMR $\left(162 \mathrm{MHz}\right.$, DMSO- $\left.d_{6}\right)$ : $\delta$ 14.4; HRMS calc. for $\mathrm{C}_{19} \mathrm{H}_{19} \mathrm{BrO}_{4} \mathrm{P}$ $[\mathrm{M}+\mathrm{H}]+:$ 421.0204, found: 421.0201 .

(-)-Dimethyl (2-(4-bromophenyl)-5-methylene-4-phenyl4,5-dihydrofuran-3-yl)phosphonate (3fa). A colorless oil was obtained in $95 \%$ yield after purification with column chromatography on silica gel (hexane/ethyl acetate, 4:1-1:1). 89\% ee was determined by chiral HPLC (Chiralcel OJ-H, $n$-hexane/ $i-\mathrm{PrOH}=80 / 20,0.8 \mathrm{~mL} / \mathrm{min}, 254 \mathrm{~nm}, 40^{\circ} \mathrm{C}$ ): $t_{\mathrm{R}}$ (major) $=7.9$ $\min , t_{\mathrm{R}}$ (minor) $=6.1 \mathrm{~min} .[\alpha]_{\mathrm{D}}{ }^{24}=-80.4\left(c 1.11, \mathrm{CH}_{2} \mathrm{Cl}_{2}\right) \cdot{ }^{1} \mathrm{H}$ NMR (400 MHz, DMSO-d6): $\delta 7.84(\mathrm{~d}, J=8.5 \mathrm{~Hz}, 2 \mathrm{H}), 7.73(\mathrm{~d}, J=$ $8.5 \mathrm{~Hz}, 2 \mathrm{H}), 7.39-7.28(\mathrm{~m}, 5 \mathrm{H}), 5.08-5.02(\mathrm{~m}, 1 \mathrm{H}), 4.81-4.75$ (m, 1H), 4.20 (s, 1H), 3.40 (d, $J=11.3 \mathrm{~Hz}, 3 \mathrm{H}), 3.21$ (d, $J=11.3$ $\mathrm{Hz}, 3 \mathrm{H}$ ).; ${ }^{13} \mathrm{C}$ NMR (100 MHz, DMSO- $\left.d 6\right): \delta 164.3$ (d, $J=13.8 \mathrm{~Hz}$ ), $162.4(\mathrm{~d}, J=25.6 \mathrm{~Hz}), 142.4,131.8,131.0,129.1,128.2,127.7$, $125.3,103.5$ (d, $J=213.4 \mathrm{~Hz}), 88.2,54.7(\mathrm{~d}, J=9.9 \mathrm{~Hz}), 52.6$ (d, $J$ $=5.7 \mathrm{~Hz}$ ), 52.4 (d, $J=5.5 \mathrm{~Hz}$ ); ${ }^{31} \mathrm{P}$ NMR (162 MHz, DMSO-d6): $\delta$ 16.1; HRMS calc. for $\mathrm{C}_{19} \mathrm{H}_{19} \mathrm{BrO}_{4} \mathrm{P}[\mathrm{M}+\mathrm{H}]^{+}:$421.0204, found: 421.0202.

(-)-Dimethyl (2-(4-methoxyphenyl)-5-methylene-4-phenyl4,5-dihydrofuran-3-yl)phosp-honate (3ga). A colorless oil was obtained in $88 \%$ yield after purification with column chromatography on silica gel (hexane/ethyl acetate, 4:1-1:1). 90\% ee was determined by chiral HPLC (Chiralcel OJ-H, $n$-hexane/ $i-\mathrm{PrOH}=90 / 10,0.8 \mathrm{~mL} / \mathrm{min}, 254 \mathrm{~nm}, 40{ }^{\circ} \mathrm{C}$ ): $t_{\mathrm{R}}$ (major) $=$ $15.7 \mathrm{~min}, t_{\mathrm{R}}$ (minor) $=10.9 \mathrm{~min} .[\alpha]_{\mathrm{D}^{22}}=-74.7\left(c 1.17, \mathrm{CH}_{2} \mathrm{Cl}_{2}\right)$. ${ }^{1} \mathrm{H}$ NMR (400 MHz, DMSO-d6): $\delta 7.90(\mathrm{~d}, J=8.8 \mathrm{~Hz}, 2 \mathrm{H})$, 7.39-7.25 (m, 5H), 7.07 (d, $J=8.8 \mathrm{~Hz}, 2 \mathrm{H}), 5.05-4.96(\mathrm{~m}, 1 \mathrm{H})$, $4.80-4.71(\mathrm{~m}, 1 \mathrm{H}), 4.18(\mathrm{~s}, 1 \mathrm{H}), 3.83(\mathrm{~s}, 3 \mathrm{H}), 3.37(\mathrm{~d}, J=11.3 \mathrm{~Hz}$, $3 \mathrm{H}), 3.21(\mathrm{~d}, J=11.3 \mathrm{~Hz}, 3 \mathrm{H}) ;{ }^{13} \mathrm{C}$ NMR (100 MHz, DMSO-d $): \delta$ 169.1 (d, $J=14.0 \mathrm{~Hz}$ ), 168.1 (d, $J=26.0 \mathrm{~Hz}$ ), $166.6,147.7,135.5$, 133.8, 132.9, 132.4, 125.4, 118.9, 105.2 (d, $J=214.8 \mathrm{~Hz}$ ), 92.4, 60.6, 59.5 (d, $J=10.1 \mathrm{~Hz}), 57.2(\mathrm{~d}, J=5.7 \mathrm{~Hz}), 57.0(\mathrm{~d}, J=5.5$ $\mathrm{Hz}$ ); ${ }^{31 \mathrm{P}} \mathrm{NMR}\left(162 \mathrm{MHz}, \mathrm{DMSO}-d_{6}\right): \delta 22.0$; HRMS calc. for $\mathrm{C}_{20} \mathrm{H}_{22} \mathrm{O}_{5} \mathrm{P}[\mathrm{M}+\mathrm{H}]^{+}:$373.1205, found: 373.1204 .

(-)-Dimethyl (2-(4-chlorophenyl)-5-methylene-4-phenyl4,5-dihydrofuran-3-yl)posphonate (3ha). A colorless oil was obtained in 95\% yield after purification with column chromatography on silica gel (hexane/ethyl acetate, 4:1-1:1). $90 \%$ ee was determined by chiral HPLC (Chiralcel OJ-H, $n$-hexane/ $i-\mathrm{PrOH}=90 / 10,0.8 \mathrm{~mL} / \mathrm{min}, 254 \mathrm{~nm}, 40^{\circ} \mathrm{C}$ ): $t_{\mathrm{R}}$ (major) $=11.3$ $\min , t_{\mathrm{R}}$ (minor) $=8.1 \mathrm{~min} .[\alpha]_{\mathrm{D}}{ }^{22}=-93.6\left(c 1.00, \mathrm{CH}_{2} \mathrm{Cl}_{2}\right) \cdot{ }^{1} \mathrm{H}$ NMR (400 MHz, DMSO- $\left.d_{6}\right): \delta 7.92(\mathrm{~d}, J=8.6 \mathrm{~Hz}, 2 \mathrm{H}), 7.59(\mathrm{~d}, J=$ $8.6 \mathrm{~Hz}, 2 \mathrm{H}), 7.39-7.28(\mathrm{~m}, 5 \mathrm{H}), 5.09-5.01(\mathrm{~m}, 1 \mathrm{H}), 4.81-4.74$ (m, 1H), 4.20 (s, 1H), 3.39 (d, $J=11.3 \mathrm{~Hz}, 3 \mathrm{H}), 3.20$ (d, $J=11.3$ $\mathrm{Hz}, 3 \mathrm{H}) ;{ }^{13} \mathrm{C}$ NMR (100 MHz, DMSO- $\left.d_{6}\right): \delta 169.0(\mathrm{~d}, J=13.9 \mathrm{~Hz})$, 167.0 (d, $J=25.7 \mathrm{~Hz}$ ), 147.2, 141.1, 135.6, 133.8, 133.6, 133.0,
132.5, 132.1, $108.2(\mathrm{~d}, J=213.4 \mathrm{~Hz}), 92.9,59.4(\mathrm{~d}, J=9.9 \mathrm{~Hz})$, $57.3(\mathrm{~d}, J=5.7 \mathrm{~Hz}), 57.1$ (d, $J=5.5 \mathrm{~Hz})$; ${ }^{31} \mathrm{P}$ NMR $(162 \mathrm{MHz}$, DMSO-d6): $\delta$ 20.9; HRMS calc. for $\mathrm{C}_{19} \mathrm{H}_{19} \mathrm{ClO}_{4} \mathrm{P}[\mathrm{M}+\mathrm{H}]^{+}$: 377.0709, found: 377.0711 .

(-)-Dimethyl (5-methylene-4-phenyl-4,5-dihydro-[2,2'-bifuran]-3-yl)phosphonate (3ia). A colorless oil was obtained in $90 \%$ yield after purification with column chromatography on silica gel (hexane/ethyl acetate, 4:1-1:1). 89\% ee was determined by chiral HPLC (Chiralcel $0 \mathrm{~J}-\mathrm{H}, n$-hexane $/ i-\mathrm{PrOH}=$ $70 / 30,0.8 \mathrm{~mL} / \mathrm{min}, 254 \mathrm{~nm}, 40{ }^{\circ} \mathrm{C}$ ): $t_{\mathrm{R}}$ (major) $=11.5 \mathrm{~min}, t_{\mathrm{R}}$ (minor) $=5.6 \mathrm{~min} .[\alpha]_{\mathrm{D}}{ }^{24}=-146.8\left(c 0.55, \mathrm{CH}_{2} \mathrm{Cl}_{2}\right) .{ }^{1} \mathrm{H} \mathrm{NMR}$ (400 MHz, DMSO- $\left.d_{6}\right): \delta 8.00-7.96(\mathrm{~m}, 1 \mathrm{H}), 7.40-7.34(\mathrm{~m}, 3 \mathrm{H})$, 7.28-7.25 (m, 3H), 6.74-6.73 (m, 1H), 5.06-5.01 (m, 1H), 4.80-4.75 (m, 1H), 4.19 (s, 1H), 3.48 (d, J= 11.4 Hz, 3H), $3.28(\mathrm{~d}$, $J=11.4 \mathrm{~Hz}, 3 \mathrm{H}) ;{ }^{13} \mathrm{C}$ NMR (100 MHz, DMSO- $\left.d 6\right): \delta 164.1(\mathrm{~d}, J=$ $13.8 \mathrm{~Hz}$ ), 153.4 (d, $J=24.6 \mathrm{~Hz}), 146.6,142.6,130.3,129.0$, 128.1, 127.7, 116.3, 112.8, 100.6 (d, J = 214.8 Hz), 88.2, 54.2 (d, $J=9.3 \mathrm{~Hz}), 52.5(\mathrm{~d}, J=5.7 \mathrm{~Hz}), 52.4(\mathrm{~d}, J=5.4 \mathrm{~Hz})$; ${ }^{31} \mathrm{P}$ NMR $(162$ MHz, DMSO- $d_{6}$ ): $\delta$ 15.4; HRMS calc. for $\mathrm{C}_{17} \mathrm{H}_{18} \mathrm{O}_{5} \mathrm{P}[\mathrm{M}+\mathrm{H}]^{+}$: 333.0892, found: 333.0887 .

(-)-Dimethyl (2-cyclohexyl-5-methylene-4-phenyl-4,5-dihydrofuran-3-yl)phosphonate (3ja). A colorless oil was obtained in $60 \%$ yield after purification with column chromatography on silica gel (hexane/ethyl acetate, 4:1-1:1). 92\% ee was determined by chiral HPLC (Chiralcel OJ-H, $n$-hexane $/ i$-PrOH $=$ 90/10, $0.8 \mathrm{~mL} / \mathrm{min}, 254 \mathrm{~nm}, 40{ }^{\circ} \mathrm{C}$ ): $t_{\mathrm{R}}$ (major) $=6.2 \mathrm{~min}, t_{\mathrm{R}}$ (minor) $=5.0$ min. $[\alpha]_{\mathrm{D}}{ }^{24}=-134.9\left(c 1.02, \mathrm{CH}_{2} \mathrm{Cl}_{2}\right) .{ }^{1} \mathrm{H}$ NMR (400 MHz, DMSO- $\left.d_{6}\right): \delta 7.33(\mathrm{t}, J=7.4 \mathrm{~Hz}, 2 \mathrm{H}), 7.25(\mathrm{t}, J=7.3 \mathrm{~Hz}$, $1 \mathrm{H}), 7.16(\mathrm{~d}, J=7.1 \mathrm{~Hz}, 2 \mathrm{H}), 4.76-4.70(\mathrm{~m}, 1 \mathrm{H}), 4.69-4.63(\mathrm{~m}$, 1H), 4.07 (s, $1 \mathrm{H}), 3.50$ (d, $J=11.3 \mathrm{~Hz}, 3 \mathrm{H}), 3.12$ (d, $J=11.3 \mathrm{~Hz}$, $3 \mathrm{H})$; $1.88-1.64(\mathrm{~m}, 5 \mathrm{H}), 1.63-1.13(\mathrm{~m}, 6 \mathrm{H}) ;{ }^{13} \mathrm{C}$ NMR $(100$ MHz, DMSO-d6): $\delta 173.8$ (d, $J=30.0 \mathrm{~Hz}), 165.3(\mathrm{~d}, J=13.9 \mathrm{~Hz}$ ), 142.6, 128.9, 128.0, 127.5, 99.8 (d, $J=213.3 \mathrm{~Hz}$ ), 87.7, 52.5 (d, $J$ $=10.3 \mathrm{~Hz}$ ), 52.1 (d, $J=5.3 \mathrm{~Hz}$ ), 51.8 (d, $J=4.8 \mathrm{~Hz}$ ), 36.5, 30.4, 29.8, 25.9, 25.8, 25.7; ${ }^{31 P}$ NMR (162 MHz, DMSO-d6): $\delta 17.5$. HRMS calc. for $\mathrm{C}_{19} \mathrm{H}_{26} \mathrm{O}_{4} \mathrm{P}[\mathrm{M}+\mathrm{H}]^{+}:$349.1569, found:349.1569.

(-)-Dimethyl (4-(4-bromophenyl)-5-methylene-2-phenyl4,5-dihydrofuran-3-yl)phosphonate (3ba). A colorless oil was obtained in $75 \%$ yield after purification with column chromatography on silica gel (hexane/ethyl acetate, 4:1-1:1). 88\% ee was determined by chiral HPLC (Chiralcel OJ-H, $n$-hexane/ $\left.i-\mathrm{PrOH}=90 / 10,0.8 \mathrm{~mL} / \mathrm{min}, 254 \mathrm{~nm}, 40^{\circ} \mathrm{C}\right): t_{\mathrm{R}}$ (major) $=12.6$ $\min , t_{\mathrm{R}}$ (minor) $=10.2 \min .[\alpha]_{\mathrm{D}}{ }^{22}=-84.7\left(c 0.97, \mathrm{CH}_{2} \mathrm{Cl}_{2}\right) .{ }^{1} \mathrm{H}$ NMR (400 MHz, DMSO- $\left.d_{6}\right): \delta$ 7.89-7.87 $(\mathrm{m}, 2 \mathrm{H}), 7.59-7.50(\mathrm{~m}$, $5 \mathrm{H}), 7.28(\mathrm{~d}, J=8.4 \mathrm{~Hz}, 2 \mathrm{H}), 5.12-5.06(\mathrm{~m}, 1 \mathrm{H}), 4.82-4.76(\mathrm{~m}$, 1H), 4.21 (s, 1H), 3.40 (d, $J=11.3 \mathrm{~Hz}, 3 \mathrm{H}$ ), 3.27 (d, $J=11.3 \mathrm{~Hz}$, $3 \mathrm{H}) ;{ }^{13} \mathrm{C}$ NMR (100 MHz, DMSO- $\left.d_{6}\right): \delta 164.0(\mathrm{~d}, J=14.0 \mathrm{~Hz}$ ), 163.6, 142.0, 132.0, 131.7, 130.5, 129.0, 128.8, 128.4, 120.8, $102.2(\mathrm{~d}, J=214.3 \mathrm{~Hz}$ ), 88.3, 54.0 (d, $J=10.1 \mathrm{~Hz}$ ), 52.6 (d, $J=5.8$ $\mathrm{Hz}$ ), 52.4 (d, $J=5.6 \mathrm{~Hz}$ ). ${ }^{31} \mathrm{P}$ NMR (162 MHz, DMSO- $d_{6}$ ): $\delta$ 16.3; HRMS calc. for $\mathrm{C}_{19} \mathrm{H}_{19} \mathrm{BrO}_{4} \mathrm{P} \quad[\mathrm{M}+\mathrm{H}]^{+}$: 421.0204, found: 421.0201.

(-)-Dimethyl (4-(4-fluorophenyl)-5-methylene-2-phenyl4,5-dihydrofuran-3-yl)phosphonate (3ca). A colorless oil was obtained in $78 \%$ yield after purification with column chromatography on silica gel (hexane/ethyl acetate, 4:1-1:1). 85\% ee was determined by chiral HPLC (Chiralcel OJ-H, $n$-hexane/ 
$\left.i-\operatorname{PrOH}=90 / 10,0.8 \mathrm{~mL} / \mathrm{min}, 254 \mathrm{~nm}, 40{ }^{\circ} \mathrm{C}\right): t_{\mathrm{R}}($ major $)=11.8$ $\min , t_{\mathrm{R}}$ (minor) $=9.3 \mathrm{~min} .[\alpha]_{\mathrm{D}}{ }^{21}=-101.9\left(c \mathrm{c} 1.00, \mathrm{CH}_{2} \mathrm{Cl}_{2}\right) \cdot{ }^{1} \mathrm{H}$ NMR (400 MHz, DMSO-d6): $\delta$ 7.90-7.88 (m, 2H), 7.56-7.49 (m, $3 \mathrm{H})$, 7.37-7.33 (m, 2H), 7.22-7.18 (m, 2H), 5.11-5.09 (m, 1H), 4.82-4.75 (m, 1H), 4.21-4.20(m, 1H), 3.39 (d, $J=11.3 \mathrm{~Hz}, 3 \mathrm{H})$, 3.25 (d, $J=11.3 \mathrm{~Hz}, 3 \mathrm{H}$ ); ${ }^{13} \mathrm{C}$ NMR (100 MHz, DMSO-d6): $\delta 164.3$ (d, $J=14.1 \mathrm{~Hz}$ ), 163.5 (d, $J=25.8 \mathrm{~Hz}), 161.8$ (d, $J=243.1 \mathrm{~Hz}$ ), 138.8, 131.7, 130.2 (d, $J=8.2 \mathrm{~Hz}$ ), 129.0, 128.7, 128.5, 115.8 (d, $J=21.5 \mathrm{~Hz}), 102.6(\mathrm{~d}, J=214.1 \mathrm{~Hz}), 88.1,53.8(\mathrm{~d}, J=10.0 \mathrm{~Hz})$, 52.6 (d, $J=5.8 \mathrm{~Hz}$ ), 52.3 (d, $J=5.5 \mathrm{~Hz}) ;{ }^{31} \mathrm{P}$ NMR $(162 \mathrm{MHz}$, DMSO- $\left.d_{6}\right): \delta 16.4$; HRMS calc. for $\mathrm{C}_{19} \mathrm{H}_{19} \mathrm{FO}_{4} \mathrm{P}[\mathrm{M}+\mathrm{H}]{ }^{+}: 361.1005$, found: 361.1003 .

(-)-Dimethyl (4-(3-chlorophenyl)-5-methylene-2-phenyl4,5-dihydrofuran-3-yl)phosphoate (3da). A colorless oil was obtained in $90 \%$ yield after purification with column chromatography on silica gel (hexane/ethyl acetate, 4:1-1:1). 81\% ee was determined by chiral HPLC (Chiralcel OJ-H, $n$-hexane/ $i-\mathrm{PrOH}=90 / 10,0.8 \mathrm{~mL} / \mathrm{min}, 254 \mathrm{~nm}, 40^{\circ} \mathrm{C}$ ): $t_{\mathrm{R}}$ (major) $=$ $10.0 \mathrm{~min}, t_{\mathrm{R}}$ (minor) $=7.8 \mathrm{~min} .[\alpha]_{\mathrm{D}}{ }^{22}=-79.9\left(c 1.10, \mathrm{CH}_{2} \mathrm{Cl}_{2}\right) .{ }^{1} \mathrm{H}$ NMR (400 MHz, DMSO- $\left.d_{6}\right): \delta 7.91-7.89(\mathrm{~m}, 2 \mathrm{H}), 7.56-7.50(\mathrm{~m}$, $3 \mathrm{H}), 7.42-7.35(\mathrm{~m}, 3 \mathrm{H}), 7.29$ (d, $J=7.5 \mathrm{~Hz}, 1 \mathrm{H}), 5.15-5.11(\mathrm{~m}$, $1 \mathrm{H})$, 4.83-4.77 (m, 1H), 4.25 (s, 1H), 3.41 (d, $J=11.3 \mathrm{~Hz}, 3 \mathrm{H})$, 3.28 (d, $J=11.3 \mathrm{~Hz}, 3 \mathrm{H}) ;{ }^{13} \mathrm{C}$ NMR (100 MHz, DMSO- $\left.d_{6}\right): \delta 163.9$ (d, $J=23.6 \mathrm{~Hz}), 163.7$ (d, $J=11.7 \mathrm{~Hz}), 145.0,133.6,131.7,131.0$, 129.0, 128.7, 128.4, 128.1, 127.7, 126.9, 102.1 (d, $J=214.4 \mathrm{~Hz}$ ), 88.5, 54.1 (d, $J=10.0 \mathrm{~Hz}$ ), 52.6 (d, $J=5.8 \mathrm{~Hz}$ ), 52.3 (d, $J=5.5$ $\mathrm{Hz}$ ); ${ }^{31 \mathrm{P}} \mathrm{NMR}$ (162 MHz, DMSO-d6): $\delta$ 16.2; HRMS calc. for $\mathrm{C}_{19} \mathrm{H}_{19} \mathrm{ClO}_{4} \mathrm{P}[\mathrm{M}+\mathrm{H}]+:$ 377.0709, found:377.0711.

(-)-Dimethyl (5-methylene-4-(naphthalen-2-yl)-2-phenyl4,5-dihydrofuran-3-yl)phosphonate (3ea). A colorless oil was obtained in $81 \%$ yield after purification with column chromatography on silica gel (hexane/ethyl acetate, 4:1-1:1). 87\% ee was determined by chiral HPLC (Chiralcel OJ-H, $n$-hexane/ $i-\mathrm{PrOH}=80 / 20,0.8 \mathrm{~mL} / \mathrm{min}, 254 \mathrm{~nm}, 40{ }^{\circ} \mathrm{C}$ ): $t_{\mathrm{R}}$ (major) $=14.3$ $\min , t_{\mathrm{R}}$ (minor) $=8.6 \mathrm{~min} .[\alpha]_{\mathrm{D}}{ }^{21}=-99.2\left(\mathrm{c} 1.02, \mathrm{CH}_{2} \mathrm{Cl}_{2}\right) \cdot{ }^{1} \mathrm{H}$ NMR (400 MHz, DMSO- $d 6): \delta 7.99-7.85(\mathrm{~m}, 6 \mathrm{H}), 7.60-7.43(\mathrm{~m}$, $6 \mathrm{H}), 5.29-5.23(\mathrm{~m}, 1 \mathrm{H}), 4.84-4.79(\mathrm{~m}, 1 \mathrm{H}), 4.23(\mathrm{~s}, 1 \mathrm{H}), 3.38(\mathrm{~d}$, $J=11.3 \mathrm{~Hz}, 3 \mathrm{H}), 3.21(\mathrm{~d}, J=11.3 \mathrm{~Hz}, 3 \mathrm{H}) ;{ }^{13} \mathrm{C}$ NMR $(100 \mathrm{MHz}$, DMSO-d6): $\delta 164.3$ (d, $J=13.9 \mathrm{~Hz}$ ), 163.7 (d, $J=25.8 \mathrm{~Hz}$ ), 139.9, $133.5,132.7,131.7,129.1,128.9,128.8,128.6,128.2,128.0$, $126.9,126.8,126.4,126.2,102.5(\mathrm{~d}, J=214.2 \mathrm{~Hz}$ ), 88.3, 54.9 (d, $J$ $=10.0 \mathrm{~Hz}), 52.8(\mathrm{~d}, J=5.8 \mathrm{~Hz}), 52.3(\mathrm{~d}, J=5.5 \mathrm{~Hz}) .{ }^{31} \mathrm{P}$ NMR $(162$ MHz, DMSO-d6): $\delta$ 16.5; HRMS calc. for $\mathrm{C}_{23} \mathrm{H}_{22} \mathrm{O}_{4} \mathrm{P}[\mathrm{M}+\mathrm{H}]^{+}$: 393.1256, found: 393.1260 .

\section{Results and discussion}

Our initial studies focused on probing the effects of different ligands, copper salts, bases and reaction temperature on the efficiency of the reaction (Table 1). 1-Phenylprop-2-yn-1-yl acetate (1a) and dimethyl (2-oxo-2-phenylethyl)phosphonate (2a) were selected as model substrates for this reaction, which was performed in the presence of $5 \mathrm{~mol} \%$ copper catalyst and 1.2 equiv. of $t$ - $\mathrm{BuOK}$ in $\mathrm{MeOH}(3 \mathrm{~mL}$ ) at room temperature for $12 \mathrm{~h}$. We first investigated a variety of chiral ligands which were efficient in the $\mathrm{Cu}$ catalyzed asymmetric propargylic substitution and cycloaddtion reactions. However, with BINAP
Table 1

Screening the reaction conditions.
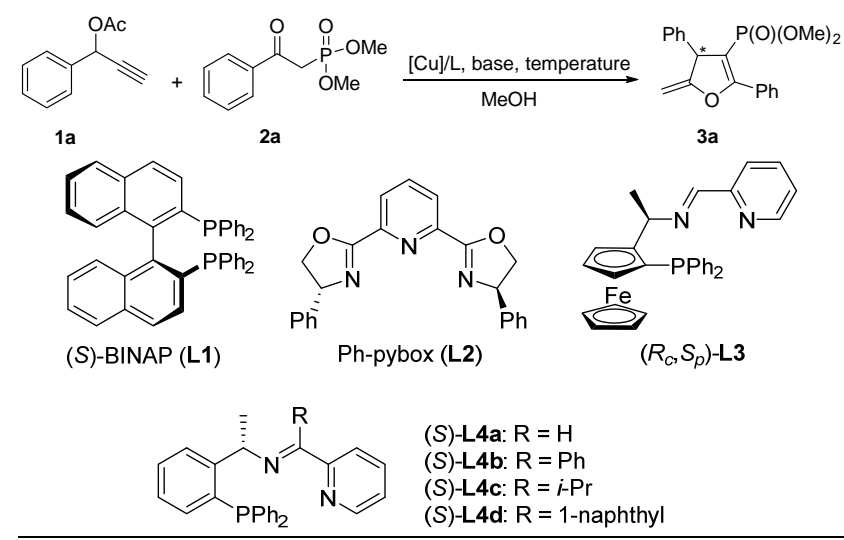

\begin{tabular}{|c|c|c|c|c|c|c|}
\hline Entry & {$[\mathrm{Cu}]$} & $\mathbf{L}$ & Base & $\begin{array}{l}\text { Temper- } \\
\text { ature }\left({ }^{\circ} \mathrm{C}\right)\end{array}$ & $\begin{array}{l}\text { Yield a }^{\text {a }} \\
(\%) \\
\end{array}$ & $\begin{array}{l}e^{b} \\
(\%)\end{array}$ \\
\hline 1 & $\mathrm{Cu}(\mathrm{OTf})_{2}$ & L1 & $t$-BuOK & $\mathrm{rt}$ & NR & $-^{c}$ \\
\hline 2 & $\mathrm{Cu}(\mathrm{OTf})_{2}$ & L2 & $t$-BuOK & $\mathrm{rt}$ & NR & $-^{c}$ \\
\hline 3 & $\mathrm{Cu}(\mathrm{OTf})_{2}$ & L3 & $t$-BuOK & $\mathrm{rt}$ & 29 & 50 \\
\hline 4 & $\mathrm{Cu}(\mathrm{OTf})_{2}$ & L4a & $t$-BuOK & $\mathrm{rt}$ & 61 & 65 \\
\hline 5 & $\mathrm{Cu}(\mathrm{OTf})_{2}$ & L4b & $t$-BuOK & $\mathrm{rt}$ & 68 & 75 \\
\hline 6 & $\mathrm{Cu}(\mathrm{OTf})_{2}$ & L4c & $t$-BuOK & $\mathrm{rt}$ & 49 & 73 \\
\hline 7 & $\mathrm{Cu}(\mathrm{OTf})_{2}$ & L4d & $t$-BuOK & $\mathrm{rt}$ & 77 & 74 \\
\hline 8 & $\mathrm{CuOAc})_{2} \cdot \mathrm{H}_{2} \mathrm{O}$ & L4b & $t$-BuOK & $\mathrm{rt}$ & 80 & 74 \\
\hline 9 & $\mathrm{CuI}$ & L4b & $t$-BuOK & $\mathrm{rt}$ & 78 & 73 \\
\hline 10 & $\mathrm{CuCl}$ & L4b & $t$-BuOK & $\mathrm{rt}$ & 80 & 58 \\
\hline 11 & $\mathrm{Cu}(\mathrm{OTf}) \cdot\left(\mathrm{C}_{6} \mathrm{H}_{6}\right)_{0.5}$ & L4b & $t$-BuOK & $\mathrm{rt}$ & 20 & 15 \\
\hline 12 & $\mathrm{Cu}\left(\mathrm{CH}_{3} \mathrm{CN}\right)_{4} \mathrm{BF}_{4}$ & L4b & $t$-BuOK & $\mathrm{rt}$ & 78 & 43 \\
\hline 13 & $\mathrm{Cu}(\mathrm{OTf})_{2}$ & L4b & none & $\mathrm{rt}$ & NR & $-^{c}$ \\
\hline 14 & $\mathrm{Cu}(\mathrm{OTf})_{2}$ & L4b & DBU & $\mathrm{rt}$ & 77 & 73 \\
\hline 15 & $\mathrm{Cu}(\mathrm{OTf})_{2}$ & L4b & $\mathrm{Cs}_{2} \mathrm{CO}_{3}$ & $\mathrm{rt}$ & 53 & 62 \\
\hline 16 & $\mathrm{Cu}(\mathrm{OTf})_{2}$ & L4b & $\mathrm{Et}_{3} \mathrm{~N}$ & $\mathrm{rt}$ & 59 & 29 \\
\hline 17 & $\mathrm{Cu}(\mathrm{OTf})_{2}$ & L4b & $i-\mathrm{Pr}_{2} \mathrm{NEt}$ & $\mathrm{rt}$ & 30 & 72 \\
\hline 18 & $\mathrm{Cu}(\mathrm{OTf})_{2}$ & L4b & $t$-BuOK & 0 & 63 & 88 \\
\hline $19 \mathrm{~d}$ & $\mathrm{Cu}(\mathrm{OTf})_{2}$ & L4b & $t$-BuOK & -20 & 88 & 90 \\
\hline
\end{tabular}

Reaction conditions: 1a $(0.6 \mathrm{mmol}), 2 a(0.3 \mathrm{mmol})$, [Cu] $(0.015 \mathrm{mmol}, 5$ $\mathrm{mol} \%)$, $\mathbf{L}(0.0165 \mathrm{mmol}, 5.5 \mathrm{~mol} \%)$ and base $(0.36 \mathrm{mmol})$ in solvent ( 3 $\mathrm{mL}$ ) for $12 \mathrm{~h}$, unless otherwise specified.

a Isolated yield.

b Determined by chiral HPLC analysis.

c Not determined because of a low conversion.

${ }^{\mathrm{d}}$ Reaction was carried out for $24 \mathrm{~h}$.

(L1) and Ph-pybox (L2) as ligands, no reaction was observed (Table 1, entries 1 and 2). Subsequent ligand screening identified chiral tridentate $\mathrm{P}, \mathrm{N}, \mathrm{N}$ ligands developed by our group as promising ligands (Table 1, entries 3-7). In particular, a bulky and structurally rigid tridentate ketamine $\mathrm{P}, \mathrm{N}, \mathrm{N}$ ligand $(S)$-L4b displayed good performance, affording the desired cycloadduct dimethyl (5-methylene-2,4-diphenyl-4,5-dihydrofuran3-yl) phosphonate (3aa) in moderate yield and enantioselectivity (Table 1, entry 5). Thus, (S)-L4b was selected as the optimal ligand for further evaluation. A variety of copper salts were next investigated. The results revealed that copper salts had an important impact on the reactivity and enantioselectivity of the reaction (Table 1, entries 8-12). These results demonstrated that $\mathrm{Cu}(\mathrm{OTf})_{2}$ was the best $\mathrm{Cu}$ source for the reaction (Table 1 , entry 5). The addition of a base was critical to the reaction since none of the desired product was observed in its absence (Table 1 , entry 13). Of the base that we tested, $t$-BuOK provided the 
Table 2

Substrate scope of $\beta$-keto phosphonates 2 .

\begin{tabular}{|c|c|c|c|c|}
\hline & O & $\begin{array}{r}\mathrm{Cu}(\mathrm{OTf})_{2}(5 \mathrm{r} \\
\mathbf{L 4 b}(5.5 \mathrm{~m}\end{array}$ & & \\
\hline 1a & 2 & $\begin{array}{r}t \text {-BuOK }(1.2 \\
\mathrm{MeOH},-20^{\circ} \mathrm{C}\end{array}$ & & \\
\hline Entry & $2\left(\mathrm{R}^{1}\right)$ & 3 & Yield a (\%) & $\mathrm{ee}^{\mathrm{b}}(\%)$ \\
\hline 1 & 2a, $\mathrm{R}^{1}=\mathrm{Ph}$ & 3аa & 88 & 90 \\
\hline 2 & $\mathbf{2 b}, \mathrm{R}^{1}=4-\mathrm{MeC}_{6} \mathrm{H}_{4}$ & 3ab & 79 & 89 \\
\hline 3 & 2c, $\mathrm{R}^{1}=3-\mathrm{MeC}_{6} \mathrm{H}_{4}$ & 3ac & 84 & 90 \\
\hline 4 & 2d, $\mathrm{R}^{1}=2-\mathrm{MeC}_{6} \mathrm{H}_{4}$ & 3ad & 61 & 88 \\
\hline 5 & $2 e, \mathrm{R}^{1}=2-\mathrm{BrC}_{6} \mathrm{H}_{4}$ & 3ae & 68 & 92 \\
\hline 6 & 2f, $\mathrm{R}^{1}=4-\mathrm{BrC}_{6} \mathrm{H}_{4}$ & 3af & 95 & 89 \\
\hline 7 & $2 \mathrm{~g}, \mathrm{R}^{1}=4-\mathrm{OMeC}_{6} \mathrm{H}_{4}$ & 3ag & 88 & 90 \\
\hline 8 & $2 h, \mathrm{R}^{1}=4-\mathrm{ClC}_{6} \mathrm{H}_{4}$ & 3ah & 95 & 90 \\
\hline 9 & $2 \mathbf{i}, \mathrm{R}^{1}=2$-furyl & 3ai & 90 & 89 \\
\hline 10 & $2 \mathbf{j}, \mathrm{R}^{1}=2$-cyclohexyl & 3aj & 60 & 92 \\
\hline
\end{tabular}

Reaction conditions: 1a $(0.6 \mathrm{mmol}), 2(0.3 \mathrm{mmol}), \mathrm{Cu}(\mathrm{OTf})_{2}(0.015$ mmol, $5 \mathrm{~mol} \%)$, (S)-L4b (0.0165 mmol, $5.5 \mathrm{~mol} \%)$ and $t$-BuOK $(0.36$ $\mathrm{mmol})$ in $\mathrm{MeOH}(3 \mathrm{~mL})$ at $-20^{\circ} \mathrm{C}$ for $24 \mathrm{~h}$.

a Isolated yields.

b Determined by chiral HPLC analysis.

best result, while DBU showed a similar result (Table 1, entries 5 and 14). Other bases such as $\mathrm{Cs}_{2} \mathrm{CO}_{3}, \mathrm{Et}_{3} \mathrm{~N}$ and $i-\mathrm{Pr}_{2} \mathrm{Nt}$ only gave a low yield or low ee value (Table 1, entries 15-17). Lowering the reaction temperature to $-20^{\circ} \mathrm{C}$ significantly improved the enantioselectivity, affording the [3+2] cycloadduct 3aa in $88 \%$ yield and with $90 \%$ ee (Table 1 , entry 19 ).

Having established the optimized conditions, we then examined the scope of the reaction using a variety of different $\beta$-keto phosphonates. The results are summarized in Table 2. The results indicated that the substitution pattern of the phenyl ring had no obvious impact on the enantioselectivity. Good enantioselectivites (88\%-92\% ee) were obtained for the $\beta$-keto phosphonates with both electron-donating and electron-withdrawing groups at the ortho, meta or para position of the phenyl ring (Table 2, entries 1-8). However, the 2-Me and 2-Br substituted substrates (2d and $\mathbf{2 e}$ ) resulted in decreased yield, presumably due to the steric hindrance (Table 2, entries 4 and 5). The 2-furyl substrate $\mathbf{2} \mathbf{i}$ also performed well in the reaction, giving the corresponding cycloadduct 3ai in $90 \%$ yield and with $89 \%$ ee (Table 2, entry 9). For the aliphatic substrate $\mathbf{2 j}$, a lower conversion was observed. The desired product 3aj was obtained in 60\% yield and $92 \%$ ee (Table 2, entry 10).

The scope of propargylic esters was also evaluated. Some representative results are shown in Fig. 1. The results revealed that the catalyst system could also be successfully applied to a variety of propargylic esters 1 . For example, 4-Br, 4-F and 3-Cl substituted phenyl propargylic esters (1b, 1c, 1d), and

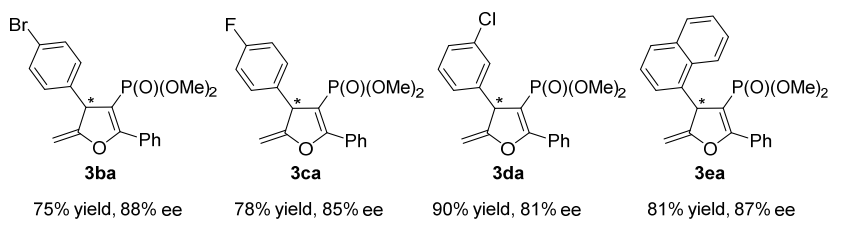

Fig. 1. Substrate scope of propargylic esters 1. 1-naphthyl substituted propargylic esters 1e all reacted smoothly under the optimized conditions to give the corresponding [3+2] cycloaddition products $\mathbf{3 b a - 3 e a ~ i n ~ g o o d ~ y i e l d s ~}$ and enantioselectivities.

\section{Conclusions}

We developed copper catalyzed asymmetric formal [3+2] cycloaddition of propargylic esters with $\beta$-keto phosphonates for the synthesis of chiral phosphonylated 2,3-dihydrofurans. By using a bulky and structurally rigid tridentate ketamine $\mathrm{P}, \mathrm{N}, \mathrm{N}$ ligand, the cycloaddition reaction proceeded smoothly with a wide range of propargylic esters and $\beta$-keto phosphonates, affording the desired optically active phosphonylated 2,3-dihydrofurans in high yields and up to $92 \%$ ee.

\section{References}

[1] B. M. Fraga, Nat. Prod. Rep., 1992, 9, 217-241.

[2] A. T. Merritt, S. V. Ley, Nat. Prod. Rep., 1992, 9, 243-287.

[3] B. H. Lipshutz, Chem. Rev., 1986, 86, 795-819.

[4] R. S. Ward, Tetrahedron 1990, 46, 5029-5041.

[5] M. M. Faul, B. E. Huff, Chem. Rev., 2000, 100, 2407-2474.

[6] M. Rueping, A. Parra, P. U. Uria, F. Besselièvre, E. Merino, Org. Lett., 2010, 12, 5680-5683.

[7] L. P. Fan, P. Li, X. S. Li, D. C. Xu, M. M. Ge, W. D. Zhu, J. W. Xie, J. Org. Chem., 2010, 75, 8716-8719.

[8] J. H. Feng, L. L. Lin, K. R. Yu, X. H. Liu, X. M. Feng, Adv. Synth. Catal., 2015, 357, 1305-1310.

[9] M. A. Calter, R. M. Phillips, C. Flaschenriem, J. Am. Chem. Soc., 2005, 127, 14566-14567.

[10] M. A. Calter, A. Korotkov, Org. Lett., 2011, 13, 6328-6330.

[11] X. W. Dou, F. R. Zhong, Y. X. Lu, Chem. Eur. J., 2012, 18, 13945-13948.

[12] F. Ozawa, A. Kubo, Y. Matsumoto, T. Hayashi, E. Nishioka, K. Yanagi, K. Moriguchi, Organometallics, 1993, 12, 4188-4196.

[13] D. A. Evans, Z. K. Sweeney, T. Rovis, J. S. Tedrow, J. Am. Chem. Soc., 2001, 123, 12095-12096.

[14] S. Son, G. C. Fu, J. Am. Chem. Soc., 2007, 129, 1046-1047.

[15] J. L. Zhou, L. J. Wang, H. Zu, X. L. Sun, Y. Tang, ACS Catal., 2013, 3, 685-688.

[16] G. Hattori, H. Matsuzawa, Y. Miyake, Y. Nishibayashi, Angew. Chem. Int. Ed., 2008, 47, 3781-3783.

[17] R. J. Detz, M. M. E. Delville, H. Hiemstra, J. H. van Maarseveen, Angew. Chem. Int. Ed., 2008, 47, 3777-3780.

[18] Y. Nishibayashi, Synthesis, 2012, 44, 489-503.

[19] C. H. Ding, X. L. Hou, Chem. Rev., 2011, 111, 1914-1937.

[20] D. Y. Zhang, X. P. Hu, Tetrahedron Lett., 2015, 56, 283-295.

[21] X. H. Hu, Z. T. Liu, L. Shao, X. P. Hu, Synthesis, 2015, 47, 913-923.

[22] C. Zhang, Y. H. Wang, X. H. Hu, Z. Zheng, J. Xu, X. P. Hu, Adv. Synth. Catal., 2012, 354, 2854-2858.

[23] F. Z. Han, F. L. Zhu, Y. H. Wang, Y. Zou, X. H. Hu, S. Chen, X. P. Hu, Org. Lett., 2014, 16, 588-591.

[24] D. Y. Zhang, F. L. Zhu, Y. H. Wang, X. H. Hu, S. Chen, C. J. Hou, X. P. Hu, Chem. Commun., 2014, 50, 14459-14462.

[25] F. L. Zhu, X. P. Hu, Chin. J. Catal., 2015, 36, 86-92.

[26] C. Zhang, Y. Z. Hui, D. Y. Zhang, X. P. Hu, RSC Adv., 2016, 6, 14763-14767.

[27] F. L. Zhu, Y. Zou, D. Y. Zhang, Y. H. Wang, X. H. Hu, S. Chen, J. Xu, X. P. Hu, Angew. Chem. Int. Ed., 2014, 53, 1410-1414. 


\title{
Graphical Abstract
}

Chin. J. Catal., 2016, 37: 1389-1395 doi: 10.1016/S1872-2067(16)62488-9

Enantioselective synthesis of chiral phosphonylated 2,3-dihydrofurans by copper catalyzed asymmetric formal [3+2] cycloaddition of propargylic esters with $\beta$-keto phosphonates

Xiushuai Chen, Chuanjin Hou*, Qing Li, Yanjun Liu*, Ruifeng Yang, Xiangping Hu*

Dalian Polytechnic University; Dalian Institute of Chemical Physics, Chinese Academy of Sciences

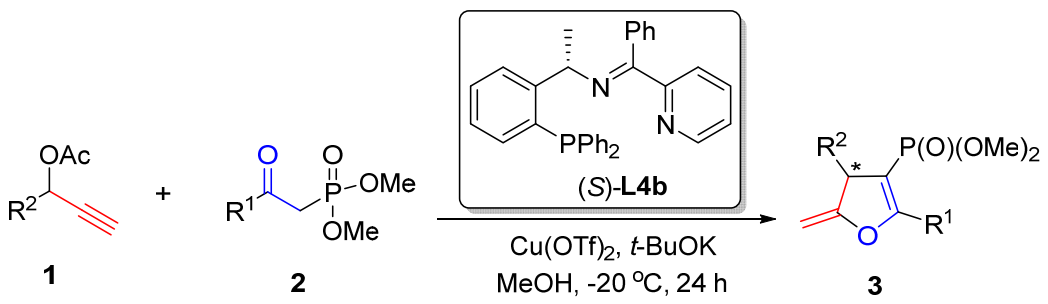

Copper catalyzed asymmetric formal [3+2] cycloaddition of propargylic esters with $\beta$-keto phosphonates for the synthesis of chiral phosphonylated 2,3-dihydrofurans was developed. By using a bulky and structurally rigid tridentate ketamine P,N,N ligand, a series of optically active phosphonylated 2,3-dihydrofurans were prepared in high yields and up to $92 \%$ ee.

[28] Y. Zou, F. L. Zhu, Z. C. Duan, Y. H. Wang, D. Y. Zhang, Z. Cao, Z. Zheng, X. P. Hu, Tetrahedron Lett., 2014, 55, 2033-2036.

[29] F. L. Zhu, Y. H. Wang, D. Y. Zhang, X. H. Hu, S. Chen, C. J. Hou, J. Xu, X. P. Hu, Adv. Synth. Catal., 2014, 356, 3231-3236.

[30] C. Zhang, X. H. Hu, Y. H. Wang, Z. Zheng, J. Xu, X. P. Hu, J. Am. Chem. Soc., 2012, 134, 9585-9588.
[31] F. L. Zhu, Y. H. Wang, D. Y. Zhang, X. P. Hu, Angew. Chem. Int. Ed., 2014, 53, 10223-10227.

[32] L. Shao, Y. H. Wang, D. Y. Zhang, J. Xu, X. P. Hu, Angew. Chem. Int. Ed., 2016, 55, 5014-5018

[33] Z. T. Liu, Y. H. Wang, F. L. Zhu, X. P. Hu, Org. Lett., 2016, 18, 1190-1193.

\section{铜催化炔丙醇酯与 $\beta$-羰基膦酸酯的不对称 $[3+2]$ 环加成反应合成 手性膦酰化2,3-二氢呋喃}

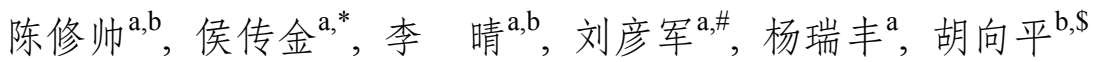 \\ ${ }^{\mathrm{a}}$ 大连工业大学轻工与化学工程学院, 辽宁大连 116034 \\ ${ }^{b}$ 中国科学院大连化学物理研究所, 辽宁大连 116023
}

摘要: 手性 2,3-二氢呋喃衍生物是一类重要的杂环化合物, 广泛存在于天然产物和生物活性分子中. 它们也经常被用于手 性四氢呋喃化合物的不对称合成. 因此, 人们发展了很多合成手性 2,3-二氢呋喃化合物的方法, 如有机小分子催化的多米 诺迈克尔-烷基化反应、“中断的” Feist-Bénary 反应或改进的 Feist-Bénary 反应. 此外, 过渡金属催化的手性 2,3-二氢呋喃 的不对称合成在近些年引起了人们的极大关注. Ozawa 等通过 Pd-催化 2,3-二氢呋喃的动力拆分方法获得了手性 2-芳基 -2,3-二氢呋喃. Evans 发展了一种 Sc-催化联烯硅和乙醛酸乙酯的 $[3+2]$ 环加成反应合成手性 2,3-二氢呋喃的方法. 最近, $\mathrm{Fu}$ 和 Tang 等发展了 $\mathrm{Cu}$ 催化烯酮和重氮化合物的[4+1]环加成反应合成手性 2,3-二氢呋喃的方法.

在 Nishibayashi 和 van Maarseveen 的开创性工作之后, $\mathrm{Cu}$ 催化的不对称炔丙基转化反应取得了很大的进展. 最近, 我 们发展了一类新的三齿手性 P,N,N-配体, 在 $\mathrm{Cu}$ 催化不对称炔丙基取代、脱羒炔丙基取代、[3+2]、[3+3]和[4+2]环加成反应 中表现出优秀的对映和非对映选择性. 其中, 我们发现采用 $\mathrm{Cu}$ 催化炔丙醇酯和 $\beta$-酮酯的 $[3+2]$ 环加成反应, 能高对映选择 性地获得手性 2,3-二氢呋喃. 我们设想, 采用 $\beta$-羰基膦酸酯代替 $\beta$-酮酯, 通过这种 $\mathrm{Cu}$ 催化 $[3+2]$ 环加成反应, 将可以合成一 类具有重要生物活性的手性膦酰化 2,3-二氢呋喃化合物. 基于这种设想, 本文使用手性 P,N,N-配体, 通过 Cu催化炔丙醇酯 与 $\beta$-羊基膦酸酯的不对称 $[3+2]$ 环加成反应, 以很好的收率和最高 $92 \%$ ee 的对映选择性获得了一系列光学活性的膦酰化 2,3-二氢呋喃化合物.

我们以炔丙醇酯 $1 \mathrm{a}$ 与 $\beta$-羊基膦酸酯 $\mathbf{2 a}$ 为标准底物, 优化了反应条件, 考察了配体、 $\mathrm{Cu}$ 盐、碱和反应温度等对反应收 率和对映选择性的影响. 我们确定了最佳的反应条件：以 $\mathbf{4 b}$ 为配体, 以 $\mathrm{Cu}(\mathrm{OTf})_{2}$ 为铜盐, 以 $t$ - $\mathrm{BuOK}$ 为碱, 以 $\mathrm{MeOH}$ 为溶 剂, $-20{ }^{\circ} \mathrm{C}$ 反应 $24 \mathrm{~h}$. 在此条件下, 我们对 $\beta$-羰基磷酸酯 2 的适用范围进行了考察. 结果表明, 各种苯基取代的 $\beta$-羰膦磷酸 酯均能得到很好的收率和对映选择性. 苯环上取代基的空间效应对反应的对映选择性影响不大, 但对反应收率影响较大, 与相应 3-取代或 4-取代底物相比较, 2-取代的底物获得的收率较低. 苯环对位取代基的电子效应对反应的影响不大, 给电 
子基或吸电子基的底物, 均得到了较好的收率和对映选择性. 杂环取代的底物同样适用于该反应, 以 $90 \%$ 的收率和 $89 \%$ ee 的对映选择性获得了相应的 [3+2]环加成产物. 对于烷基底物, 虽然反应的产率略低, 但是得到了高达 $92 \%$ ee 的产物. 此外, 我们对炔丙醇酯底物的适用范围也进行了考察. 结果表明, 该体系对于各种取代的炔丙醇酯底物均可以获得较高的 收率和良好的对映选择性.

总之, 本文发展了一种铜催化炔丙醇酯与 $\beta$-羰基膦酸酯的不对称 $[3+2]$ 环加成反应的方法, 成功合成了手性膦酰化 $2,3-$ 二氢呋喃化合物. 通过使用一个结构刚性的酮亚胺三齿 P,N,N-配体, 以很好的收率和最高 $92 \%$ ee 的对映选择性获得了一 系列光学活性的膦酰化 2,3-二氢呋喃化合物.

关键词: 铜; 不对称合成; $[3+2]$ 环加成; $\beta$-羰基膦酸酯; 膦酰化 2,3 -二氢呋喃

收稿日期: 2016-05-21. 接受日期: 2016-06-14. 出版日期: 2016-08-05.

*通讯联系人. 电子信箱: houcj@dlpu.edu.cn

\#通讯联系人. 电子信箱: yjliu65@sohu.com

\$通讯联系人. 电子信箱: xiangping@dicp.ac.cn

基金来源: 国家自然科学基金 (21403022,21572226); 辽宁省自然科学基金 (2015020194).

本文的英文电子版由Elsevier出版社在ScienceDirect上出版(http://www.sciencedirect.com/science/journal/18722067). 